\title{
Efficacy and safety of migalastat in a Japanese population: a subgroup analysis of the ATTRACT study
}

\author{
Ichiei Narita ${ }^{1} \cdot$ Toya Ohashi $^{2} \cdot$ Norio Sakai $^{3} \cdot$ Takashi Hamazaki $^{4} \cdot$ Nina Skuban $^{5} \cdot$ Jeffrey P. Castelli ${ }^{5} \cdot$ Hjalmar Lagast $^{5}$. \\ Jay A. Barth ${ }^{5}$
}

Received: 19 July 2019 / Accepted: 28 October 2019 / Published online: 30 December 2019

(c) The Author(s) 2019

\begin{abstract}
Background Fabry disease is a progressive X-linked lysosomal disorder. In this subgroup analysis of the global phase III ATTRACT study, the efficacy and safety of oral migalastat, a pharmacologic chaperone, were investigated in Japanese patients with Fabry disease.

Methods Patients were randomly assigned to receive migalastat (150 mg every other day) or to continue biweekly enzyme replacement therapy infusions (ERT; agalsidase alfa $0.2 \mathrm{mg} / \mathrm{kg}$ or agalsidase beta $1.0 \mathrm{mg} / \mathrm{kg}$ ) for 18 months followed by a 12-month open-label extension during which all patients received migalastat. End points included glomerular filtration rate (estimated and measured), left ventricular mass index (LVMi), composite clinical outcomes, leukocyte alpha-galactosidase A activity, plasma globotriaosylsphingosine (lyso- $\mathrm{Gb}_{3}$ ), and safety.

Results Data from 7 Japanese patients (migalastat, 5; ERT, 2), mean age 55 years, with high disease burden, were analyzed. All patients in the migalastat group completed the open-label comparison and extension periods. At 18 months, efficacy in the Japanese patient population was similar to that in the overall ATTRACT population. Migalastat treatment increased leukocyte alpha-galactosidase A activity, stabilized renal function, and decreased LVMi. Plasma lyso- $\mathrm{Gb}_{3}$ levels remained low and stable. Additionally, the long-term extension study showed that efficacy of migalastat was maintained for up to 48 months. Migalastat was safe and well tolerated in the Japanese patients, as in the overall ATTRACT population.

Conclusion Migalastat can be used to treat Japanese patients with Fabry disease with GLA mutations amenable to migalastat according to the dosage and administration approved in other countries.
\end{abstract}

Trial registration numbers ClinicalTrials.gov, NCT01218659 and NCT02194985.

Keywords Fabry disease $\cdot$ Migalastat $\cdot$ Alpha-galactosidase $\cdot$ Pharmacologic chaperone $\cdot$ Mutation $\cdot$ Enzyme replacement therapy

Jay A. Barth

jbarth@amicusrx.com

1 Division of Clinical Nephrology and Rheumatology, Niigata University Graduate School of Medical and Dental Sciences, Niigata, Japan

2 Division of Gene Therapy, Research Center for Medical Sciences, The Jikei University School of Medicine, Tokyo, Japan

3 Division of Health Science, Osaka University Graduate School of Medicine, Osaka, Japan

4 Department of Pediatrics, Osaka City University Graduate School of Medicine, Osaka, Japan

5 Amicus Therapeutics, Inc., 1 Cedar Brook Drive, Cranbury, NJ 08512, USA

\section{Introduction}

Fabry disease is a rare progressive $\mathrm{X}$-linked lysosomal disorder, in which mutations of the GLA gene impair the activity of the lysosomal enzyme alpha-galactosidase A ( $\alpha$-Gal A), resulting in a devastating condition [1]. In Fabry disease, accumulation of substrates of $\alpha-\mathrm{Gal} \mathrm{A}$, such as globotriaosylceramide (GL-3) and globotriaosylsphingosine (lyso$\mathrm{Gb}_{3}$ ), in various organs and tissues causes dysfunction that can lead to premature death. Cardiac complications are the primary cause of death in men and women both, although some data indicate that the primary cause of death is renal complications in men and cerebrovascular disease in women $[2,3]$. The estimated prevalence of Fabry disease ranges between 1 in 476,000 and 1 in 117,000 worldwide, although 
its actual prevalence is thought to be higher [3]. In Japan, the estimated prevalence is 1 in 7000 newborns based on the results of neonatal screening [4].

Phenotypic expression of Fabry disease is highly variable. Initial symptoms of the classic disease generally appear in childhood, and symptoms progress if the condition is not treated [3]. Enzyme replacement therapy (ERT) with agalsidase alfa or agalsidase beta is the mainstay of treatment [5]. Results of previous clinical studies have shown efficacy and good tolerability of ERT in some Japanese patients with Fabry disease [6, 7]. However, several challenges remain, including infusion-associated reactions, reduced quality of life associated with lifelong parenteral treatment, and the decrease in efficacy after development of circulating antibodies to the enzyme $[8,9]$.

Migalastat is a low-molecular-weight iminosugar that can act as a pharmacologic chaperone by binding selectively and reversibly to the active site of specific mutant forms of $\alpha$-Gal A, the genotype of which is referred to as amenable GLA mutations. Migalastat binds to mutant forms of $\alpha-\mathrm{Gal}$ A in the endoplasmic reticulum and promotes trafficking to the lysosomes, increasing lysosomal enzyme activity [10-12]. The efficacy of migalastat has been verified in patients with amenable mutations [13]. Migalastat was discovered in Japan [14] and was approved for the treatment of patients with Fabry disease 16 years or older in Japan, [16] Australia, Europe, Israel, South Korea and Switzerland and in adult patients in Canada and the United States [10, 15].

A phase I pharmacokinetic study showed similar doseproportional pharmacokinetics and a similar safety profile of migalastat in Japanese and non-Japanese populations [17]. The phase III ATTRACT study compared efficacy and safety of migalastat with ERT in patients with Fabry disease with amenable mutations who were previously treated with ERT [18]. During an 18-month treatment period, migalastat and ERT both had a similar effect on renal function. From baseline to month 18 , the left ventricular mass index (LVMi) decreased significantly in the migalastat group, but there was no significant decrease in the ERT group [18]. We report the results of analyses performed in the Japanese subgroup, including data from the open-label extension study (OLE).

\section{Methods}

\section{Patients and study design}

ATTRACT was a global, open-label, randomized trial with a 30-month treatment period (18-month openlabel comparison of migalastat with ERT, and 12-month OLE with migalastat; AT1001-012, ClinicalTrials.gov, NCT01218659) in patients with Fabry disease who were previously treated with ERT. Full methods are described in the primary paper [18]. Primary inclusion criteria were men and women between 16 and 74 years of age with Fabry disease and a migalastat-amenable GLA mutation, as detected by the preliminary human embryonic kidney (HEK) 293 cell-based assay [19]. Other inclusion criteria were initiation of ERT $\geq 12$ months before the baseline visit, maintenance of a stable ERT dose for $\geq 3$ months before baseline assessment (at least $80 \%$ of the dose specified in the package insert), and an estimated glomerular filtration rate $(\mathrm{eGFR}) \geq 30 \mathrm{~mL} / \mathrm{min} / 1.73 \mathrm{~m}^{2}$ (calculated by the modification of diet in renal disease equation) at screening. In patients receiving an angiotensin-converting enzyme inhibitor, angiotensin II receptor blocker, or renin inhibitor, doses of these drugs had to be stable for $\geq 4$ weeks before screening.

Eligible patients were randomly assigned 1.5:1 using interactive response technology (Almac Clinical Technologies, Craigavon, UK) to receive either oral migalastat hydrochloride (150 mg every other day) or continue previous ERT (biweekly infusions of either agalsidase alfa $0.2 \mathrm{mg} / \mathrm{kg}$ or agalsidase beta $1.0 \mathrm{mg} / \mathrm{kg}$ ) during the 18-month open-label treatment period [18]. Patients who completed the open-label comparison period could continue treatment with migalastat (150 mg every other day) for an additional 12 months. During this OLE period, patients in the migalastat group continued to receive migalastat, whereas patients in the ERT group discontinued ERT and switched to migalastat. Patients who completed both parts of the ATTRACT study were eligible for enrollment in the OLE study (AT1001-042, ClinicalTrials. gov, NCT02194985).

\section{End points}

Coprimary end points of the ATTRACT study were the annual rate of change from baseline in eGFR calculated using the chronic kidney disease epidemiology collaboration equation (eGFR $\left.\mathrm{CKD}_{\text {-EPI }}\right)$ and measured GFR determined by clearance of iohexol ( $\left.\mathrm{mGFR}_{\text {iohexol }}\right)$ [18]. Key secondary end points were changes from baseline in LVMi and composite clinical outcome (renal, cardiac, cerebrovascular events, and death). Other secondary end points were leukocyte $\alpha$-Gal A activity, plasma lyso-Gb3 level, and safety.

The efficacy analysis was performed in the modified intention-to-treat population, which included all randomly assigned patients who received at least 1 dose of study drug and had baseline and postbaseline GFR measurements, excluding patients with $G L A$ mutations later found nonamenable in a new and good laboratory practice-validated HEK assay [13]. The safety population included all randomly assigned patients who received $\geq 1$ dose of the study medication. 


\section{Statistical analysis}

Leukocyte $\alpha$-Gal A activity was measured at baseline, months 1 and 3 , and every 3 months thereafter up to month 30. Annualized rates of change in eGFR $\mathrm{CKD}_{\text {-EPI }}$ and mGFR $_{\text {iohexol }}$ were analyzed by analysis of covariance, considering the treatment group, sex, age, baseline GFR, and baseline 24-h urine protein excretion as covariates. Descriptive statistics, including least squares mean values and $95 \%$ confidence intervals (CIs), were determined with this model. Annualized rates of change of GFR were calculated from the slopes obtained by linear regression. LVMi was evaluated centrally (Cardiocore, Rockville, MD, USA) by 2-dimensional or M-mode echocardiography in a blinded manner every 6 months. The long-term effect of migalastat on LVMi was evaluated by calculating the change from baseline to final assessment in each patient. Composite clinical outcome was evaluated based on the number of patients who experienced a prespecified renal event, cardiac event, cerebrovascular event or who died. The plasma level of lyso- $\mathrm{Gb}_{3}$ was quantified by liquid chromatography-mass spectrometry at baseline and months $6,12,18$, and 30 .

Safety was evaluated based on the type, frequency, and severity of adverse events (AEs), as well as changes in vital signs, laboratory data, and physical findings. AEs were coded according to the Medical Dictionary for Regulatory Activities (MedDRA version 8.0 or more recent versions).

\section{Results}

\section{Patients}

The ATTRACT study cohort included 60 patients initially randomly assigned to receive migalastat $(n=36)$ or to continue ERT $(n=24)$ for the first 18-month open-label comparison period. Three patients randomly assigned to the ERT group discontinued the study before the first dose; therefore, 57 patients (36 in the migalastat group and 21 in the ERT group) were included in the primary analysis. These 57 patients were reported to be amenable to migalastat based on the preliminary HEK cell-based assay. A new, good laboratory practice-validated HEK cell-based assay became available during the study and confirmed that 53 of these 57 patients had migalastat-amenable mutations [18].

The shortage of agalsidase beta in Japan between 2009 and 2012 made it difficult to find patients who met the enrollment criteria for the ATTRACT study (i.e., $\geq 12$ months of ERT with stable dosing) [20]. Despite this, 7 patients ( $>10 \%$ of the ATTRACT population) were enrolled from participating medical institutions throughout Japan. Five patients (3 men and 2 women, Table 1 ) were randomly assigned to the migalastat group and 2 patients ( 2 women) were randomly assigned to the ERT group (Fig. 1). Three patients had mutations associated with the classic phenotype of Fabry disease, and 3 had mutations associated with the late-onset phenotype. One patient randomly assigned to the ERT group had nonamenable mutation (R342Q) and was discontinued from the study after month 18; this patient was not included in the efficacy analyses. The other patient randomly assigned to the ERT group was withdrawn by the investigator at completion of the study due to deterioration of chronic heart failure. All 5 patients randomly assigned to the migalastat group completed the 30-month ATTRACT study and were then enrolled in the AT1001-042 OLE study. Of these, 1 patient subsequently was withdrawn from the OLE study by the investigator due to reduced renal function; the other 4 patients continued the OLE study (migalastat treatment duration $>44$ months).
Table 1 Baseline characteristics of the Japanese patient population and the overall patient population of the ATT RACT study (mITT population)

\begin{tabular}{|c|c|c|c|c|c|c|}
\hline \multirow[t]{2}{*}{ Item } & \multicolumn{3}{|c|}{ Japanese patient population } & \multicolumn{3}{|c|}{ Overall patient population } \\
\hline & Migalastat $(n=5)$ & $\operatorname{ERT}(n=1)$ & All $(n=6)$ & Migalastat $(n=34)$ & $\operatorname{ERT}(n=18)$ & All $(n=52)$ \\
\hline $\begin{array}{l}\text { Age, years, } \\
\text { mean } \\
(\mathrm{SD})\end{array}$ & $51.8(4.8)$ & 70.0 & $54.8(8.6)$ & $51.2(13.2)$ & $44.8(14.7)$ & $49.0(14.0)$ \\
\hline \multicolumn{7}{|l|}{ Sex, $n(\%)$} \\
\hline Male & $3(60.0)$ & 0 & $3(50.0)$ & $14(41.2)$ & $8(44.4)$ & $22(42.3)$ \\
\hline Female & $2(40.0)$ & $1(100.0)$ & $3(50.0)$ & $20(58.8)$ & $10(55.6)$ & $30(57.7)$ \\
\hline \multicolumn{7}{|l|}{ Race, $n(\%)$} \\
\hline White & 0 & 0 & 0 & $28(82.4)$ & $17(94.4)$ & $45(86.5)$ \\
\hline Asian & $5(100.0)$ & $1(100.0)$ & $6(100.0)$ & $5(14.7)$ & $1(5.6)$ & $6(11.5)$ \\
\hline Other & 0 & 0 & 0 & $1(2.9)$ & 0 & $1(1.9)$ \\
\hline
\end{tabular}

$E R T$ enzyme replacement therapy, $m I T T$ modified intention-to-treat population; $S D$, standard deviation Patients in the mITT population are those with amenable GLA mutations who were assessed for baseline and postbaseline efficacy measures 
Fig. 1 Patient flowchart: ATT RACT study. ERT enzyme replacement therapy

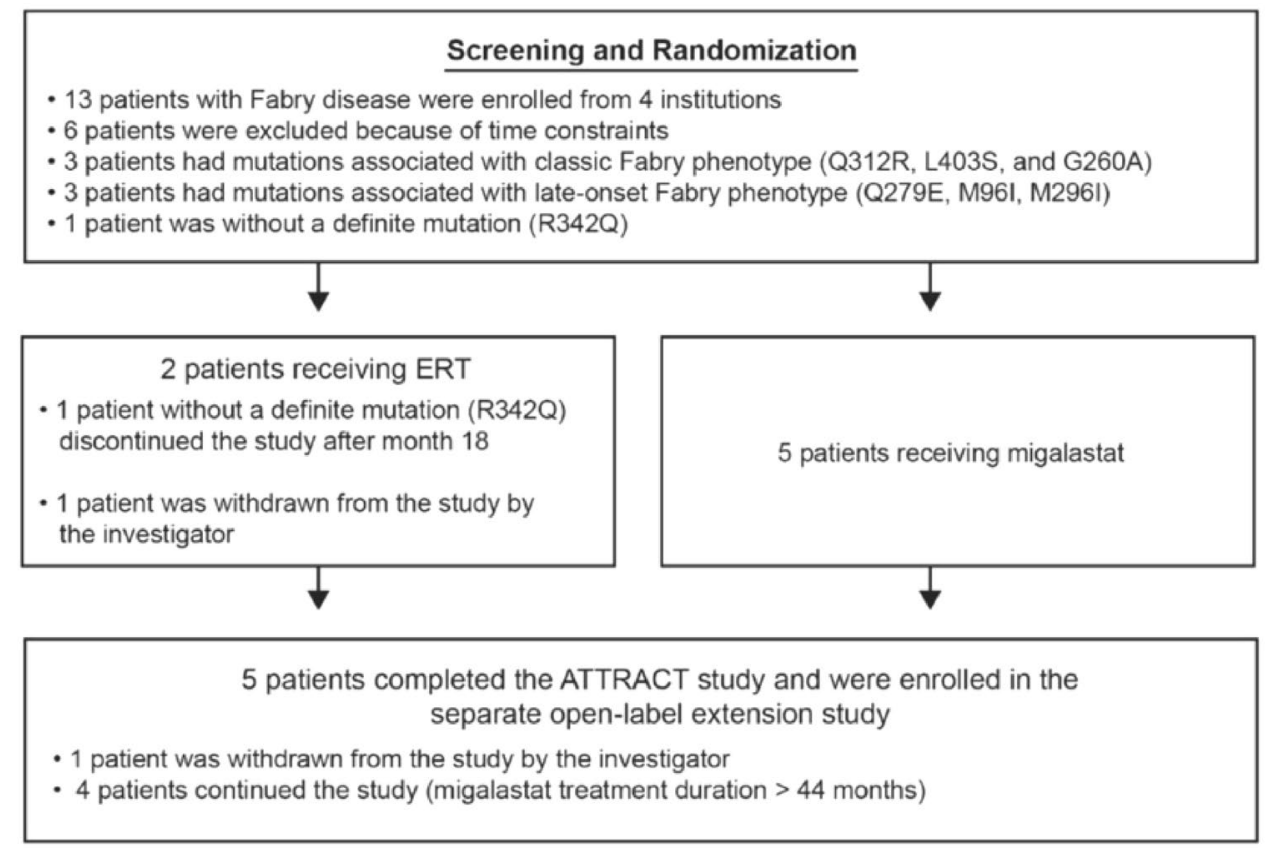

The Japanese patient population was similar to the overall patient population of the ATTRACT study (Table 1), but the mean age was slightly older (55 vs 49 years, respectively). There was an equal number of male and female patients in both patient populations.

\section{Efficacy}

Table 2 shows data on the primary and secondary end points in the Japanese patient population.

\section{Leukocyte a-Gal A activity}

Three Japanese men receiving migalastat showed an increase in leukocyte $\alpha$-Gal A activity from baseline to month 18 (change from baseline: $0.74 \mathrm{nmol} / \mathrm{h} / \mathrm{mg}, 2.25 \mathrm{nmol} / \mathrm{h} / \mathrm{mg}$, and $6.86 \mathrm{nmol} / \mathrm{h} / \mathrm{mg}$; percentage change: $50 \%, 183 \%$, and $406 \%$ ). In all men with amenable mutations in the migalastat group of the ATTRACT study $(n=14$, including 3 Japanese men), the median change in leukocyte $\alpha$-Gal A activity during the same period was $6.6 \mathrm{nmol} / \mathrm{h} / \mathrm{mg}$. In contrast, patients in the ERT group showed no change in leukocyte $\alpha$-Gal A activity from baseline (median change, $0.04 \mathrm{nmol} / \mathrm{h} / \mathrm{mg}$ ). Because women express both the mutant and the wild-type forms of $\alpha$-Gal A, measurement of leukocyte $\alpha$-Gal A activity in the female patients was considered inappropriate.

\section{Renal function}

In the overall patient population, migalastat and ERT showed a similar effect on renal function during the 18-month treatment period [17]. The mean annualized rate of change

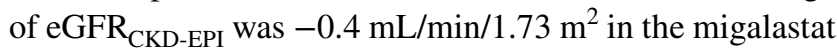
group $(n=34)$ and $-1.0 \mathrm{~mL} / \mathrm{min} / 1.73 \mathrm{~m}^{2}$ in the ERT group $(n=18)$. The mean annualized rate of change of $\mathrm{mGFR}_{\text {iohexol }}$ was $-4.4 \mathrm{~mL} / \mathrm{min} / 1.73 \mathrm{~m}^{2}$ in the migalastat group $(n=34)$ and $-3.2 \mathrm{~mL} / \mathrm{min} / 1.73 \mathrm{~m}^{2}$ in the ERT group $(n=18)$ [18].

In the Japanese patient subpopulation, the mean annualized rate of change in $\mathrm{eGFR}_{\mathrm{CKD} \text {-EPI }}$ from baseline to month 18 was $-1.8 \mathrm{~mL} / \mathrm{min} / 1.73 \mathrm{~m}^{2}$ for the migalastat group $(n=5)$ and $1.6 \mathrm{~mL} / \mathrm{min} / 1.73 \mathrm{~m}^{2}$ for the ERT group $(n=1)$ (Fig. 2a). Annualized rates of change in the 5 patients from the migalastat group ranged from $-6.97 \mathrm{~mL} / \mathrm{min} / 1.73 \mathrm{~m}^{2}$ to $2.25 \mathrm{~mL} / \mathrm{min} / 1.73 \mathrm{~m}^{2}$. The mean annualized rate of change of eGFR ${ }_{\text {CKD-EPI }}$ in the Japanese patient population was within the $95 \%$ CI of the least squares mean for the overall patient population.

In the 5 patients who received migalastat from months 18 to 30 after completion of the open-label comparison period, renal function was stable through month 30 [mean \pm standard deviation (SD) annualized rate of change in eGFR ${ }_{\mathrm{CKD} \text {-EPI }}$ from baseline to month $30:-2.94 \pm 2.93 \mathrm{~mL} / \mathrm{min} / 1.73 \mathrm{~m}^{2}$ ]. In addition, renal function was stable up to month 48 in the AT1001-042 OLE study (Fig. 2b).

In the Japanese patient subpopulation, the mean annualized rate of change in mGFR $_{\text {iohexol }}$ from baseline to month 18 was $-7.6 \mathrm{~mL} / \mathrm{min} / 1.73 \mathrm{~m}^{2}$ for the migalastat group $(n=5)$ and $-6.4 \mathrm{~mL} / \mathrm{min} / 1.73 \mathrm{~m}^{2}$ for the ERT group $(\mathrm{n}=1)$. In the 5 patients from the migalastat group, the annualized rate of change in $\mathrm{mGFR}_{\text {iohexol }}$ ranged from $-16.3 \mathrm{~mL} /$ $\mathrm{min} / 1.73 \mathrm{~m}^{2}$ to $-1.1 \mathrm{~mL} / \mathrm{min} / 1.73 \mathrm{~m}^{2}$ (Fig. 2c). This range was within the $95 \% \mathrm{CI}$ of the least squares mean for the 
Table 2 Individual patient characteristics and results for primary and key secondary end points [18-month comparison period $+12-$ month extension period (30 months of treatment)]

\begin{tabular}{|c|c|c|c|c|c|c|c|}
\hline \multirow{2}{*}{$\begin{array}{l}\text { Group } \\
\text { Subject no. }\end{array}$} & \multicolumn{5}{|c|}{ Migalastat-migalastat $(n=5)$} & \multirow{2}{*}{$\begin{array}{l}\text { ERT- } \\
\text { migalastat } \\
(n=2) \\
\text { Subject } 6^{\mathrm{b}}\end{array}$} & \multirow[b]{2}{*}{ Subject 7} \\
\hline & Subject 1 & Subject 2 & Subject 3 & Subject 4 & Subject 5 & & \\
\hline Genotype & Q312R & L403S & Q279E & M296I & M96I & $\mathrm{R} 342 \mathrm{Q}$ & G260A \\
\hline Age, years & 48 & 56 & 57 & 52 & 46 & 53 & 70 \\
\hline Sex & Male & Male & Female & Female & Male & Female & Female \\
\hline Duration of disease, years & 19.74 & 4.63 & 4.78 & 17.31 & 4.20 & 10.73 & 26.82 \\
\hline ACEI/ARB use & Yes & Yes & No & Yes & Yes & No & No \\
\hline \multicolumn{8}{|l|}{$\mathrm{eGFR}_{\text {CKD-EPI }}\left(\mathrm{mL} / \mathrm{min} / 1.73 \mathrm{~m}^{2}\right)$} \\
\hline Baseline & 51.33 & 99.74 & 97.73 & 108.34 & 54.91 & 106.85 & 44.83 \\
\hline Change ( $0-18$ months of administration) & -11.93 & 0 & -6.99 & -2.14 & -2.85 & -7.43 & 4.97 \\
\hline Annualized rate of change ( $0-18$ months of administration) & -6.97 & 2.25 & -4.25 & 1.53 & -1.48 & -1.77 & 1.56 \\
\hline Change (0/18-30 months ${ }^{\mathrm{a}}$ of administration) & -17.66 & 0.58 & -8.65 & -3.48 & -6.26 & - & -17.60 \\
\hline Annualized rate of change $\left(0 / 18-30\right.$ months $^{\mathrm{a}}$ of administration $)$ & -5.82 & 0.10 & -6.26 & -0.73 & -1.98 & - & -20.26 \\
\hline \multicolumn{8}{|l|}{$\mathrm{mGFR}_{\text {iohexol }}\left(\mathrm{mL} / \mathrm{min} / 1.73 \mathrm{~m}^{2}\right)$} \\
\hline Baseline & 56.20 & 99.80 & 107.60 & 106.80 & 52.80 & 108.00 & 33.00 \\
\hline Change (0-18 months of administration) & -10.50 & -20.00 & -25.80 & -1.10 & -1.70 & -4.20 & $-6.30^{\mathrm{c}}$ \\
\hline Annualized rate of change ( $0-18$ months of administration) & -7.22 & -11.40 & -16.32 & -1.93 & -1.11 & -4.70 & $-6.42^{\mathrm{c}}$ \\
\hline Change (0/18-30 months ${ }^{\mathrm{a}}$ of administration) & -15.10 & -13.10 & -0.40 & -5.40 & -5.60 & - & - \\
\hline Annualized rate of change ( $0 / 18-30$ months $^{\mathrm{a}}$ of administration) & -6.02 & -5.85 & -0.11 & -2.27 & -2.68 & - & - \\
\hline \multicolumn{8}{|l|}{ Left ventricular mass index $\left(\mathrm{g} / \mathrm{m}^{2}\right)$} \\
\hline Baseline & 102.64 & 165.73 & 87.83 & 74.83 & 125.63 & 122.49 & 85.34 \\
\hline Change ( $0-18$ months of administration) & -7.74 & -26.23 & 1.63 & -8.72 & -12.59 & -23.97 & 12.54 \\
\hline Change (0/18-30 months ${ }^{\mathrm{a}}$ of administration) & -17.96 & -24.46 & -0.12 & -18.86 & -5.13 & - & 13.26 \\
\hline \multicolumn{8}{|l|}{$\alpha$-Gal A activity in PBMC (nmol/h/mg) } \\
\hline Baseline & 1.69 & 1.48 & 22.42 & 17.61 & 1.23 & 11.91 & 13.64 \\
\hline Change ( $0-18$ months of administration) & 6.86 & 0.74 & 5.07 & 13.06 & 2.25 & 3.57 & -6.31 \\
\hline Change $\left(0 / 18-30\right.$ months $^{\mathrm{a}}$ of administration $)$ & 3.48 & 1.02 & 13.44 & 15.15 & 2.86 & - & 16.98 \\
\hline \multicolumn{8}{|l|}{ Plasma lyso- $\mathrm{Gb}_{3}$ level (nmol/L) } \\
\hline Baseline & 2.247 & 11.500 & 3.220 & 1.380 & 14.500 & 13.13 & 10.93 \\
\hline Change (0-18 months of administration) & 0.767 & -1.107 & 0.277 & 0.550 & -0.200 & -2.37 & $-0.57^{\mathrm{c}}$ \\
\hline Change (0/18-30 months ${ }^{\mathrm{a}}$ of administration) & 1.050 & -2.127 & 0.967 & 0.733 & -0.833 & - & - \\
\hline
\end{tabular}

$\alpha$-Gal A alpha-galactosidase A, ACEI angiotensin-converting enzyme inhibitor, ARB angiotensin II receptor blocker; $H E K$ human embryonic kidney, eGFR ${ }_{\mathrm{CKD}-E P I}$ estimated glomerular filtration rate calculated by the Chronic Kidney Disease Epidemiology Collaboration equation, ERT enzyme replacement therapy, lyso- $G b_{3}$ globotriaosylsphingosine, $m G F R_{\text {iohexol }}$ estimated glomerular filtration rate determined from clearance of iohexol, IITT modified intention-to-treat population, $P B M C$ peripheral blood mononuclear cell

${ }^{\mathrm{a}} 0 / 18-30$ months: the change or annualized rate of change from baseline in the migalastat-migalastat group, or the change or annualized rate of change from month 18 in the ERT-migalastat group

${ }^{\mathrm{b}}$ The subject was identified as having a GLA gene mutation not amenable to migalastat based on the final HEK cell-based assay and was excluded from the mITT population

${ }^{\mathrm{c}}$ Month 12

overall patient population. In addition, $\mathrm{mGFR}_{\text {iohexol }}$ was stable through month 30 (mean \pm SD annualized rate of change of $\mathrm{mGFR}_{\text {iohexol }}$ from baseline to month $30,-3.39 \pm 2.52 \mathrm{~mL} /$ $\min / 1.73 \mathrm{~m}^{2}$ ).

\section{Cardiac function}

In the overall patient population, a significant reduction in the mean of LVMi from baseline to month 18 was noted among patients who switched from ERT to migalastat at the beginning of the study $\left(-6.6 \mathrm{~g} / \mathrm{m}^{2} ; 95 \% \mathrm{CI}-11.0\right.$ to -2.2 ; $n=33$ ). No significant change was noted in patients who continued ERT $\left(-2.0 \mathrm{~g} / \mathrm{m}^{2} ; 95 \%\right.$ CI -11.0 to $\left.7.0 ; n=16\right)$ [17]. Patients who received migalastat and had baseline left ventricular hypertrophy $(n=13)$ showed the largest changes in LVMi [18].

In the Japanese patient subpopulation, the mean LVMi was reduced from baseline to month 18 in the migalastat 


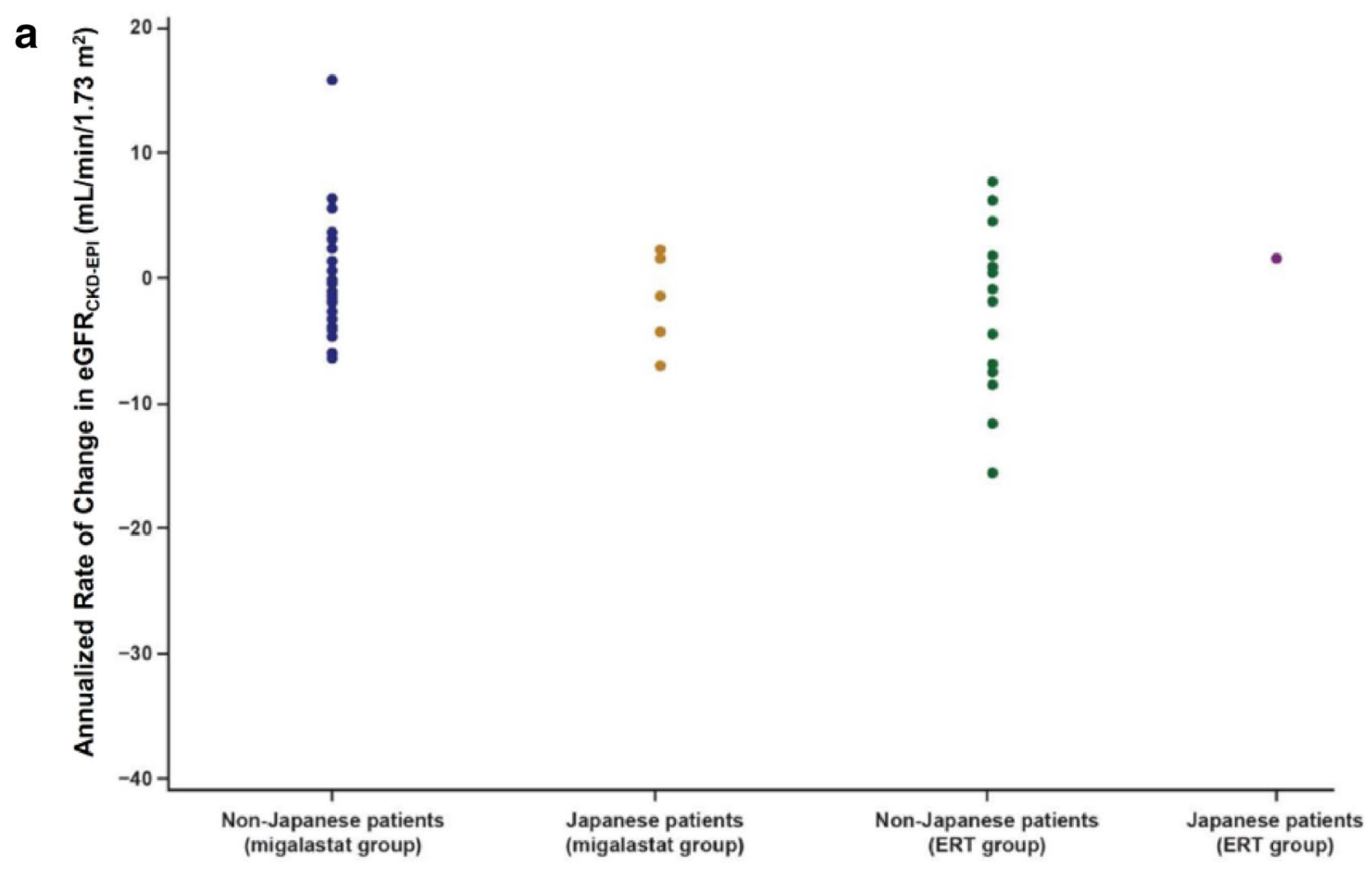

Patient Population
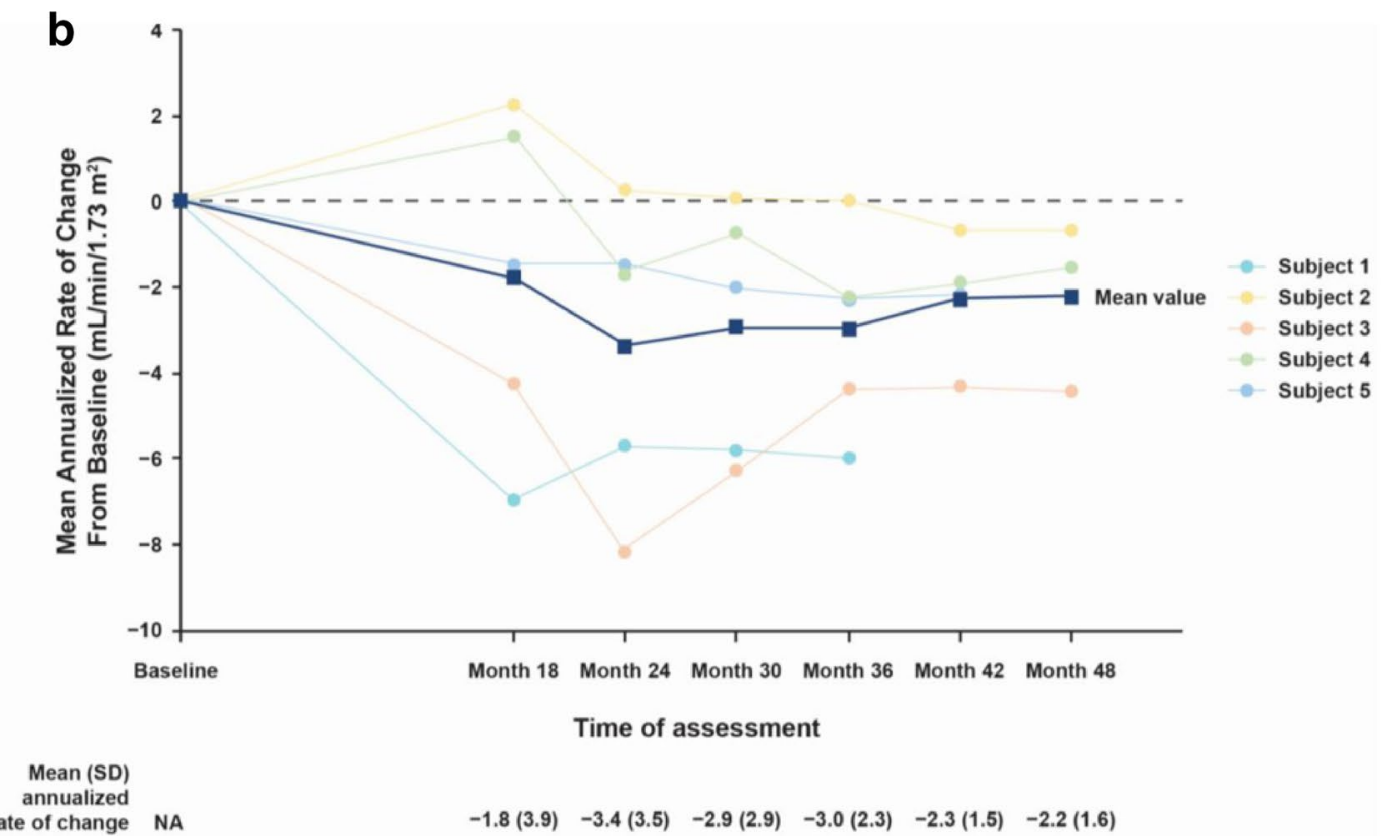

$-1.8(3.9) \quad-3.4(3.5) \quad-2.9(2.9) \quad-3.0(2.3) \quad-2.3(1.5) \quad-2.2(1.6)$

Fig. 2 Annualized rate of change in the glomerular filtration rate $\left(\mathrm{mL} / \mathrm{min} / 1.73 \mathrm{~m}^{2}\right)$. a Annualized rate of change of eGFR $\mathrm{CKD}_{\mathrm{EPI}}$ from baseline to month 18 . Dots are individual patient data. b Annualized rate of change of eGFR $\mathrm{CKD}_{\text {-EPI }}$ from baseline to month 48 in the 5 Japanese patients randomly assigned to the migalastat group at study entry. Because 1 patient was withdrawn from the study by the investigator at month 30 or thereafter, data for month 42 and month 48 are from the remaining 4 patients. Blue line is mean change from baseline; remaining lines represent individual patient data. c Annualized rate of change of $\mathrm{mGFR}_{\text {iohexol }}$ from baseline to month 18 . $e G F R_{C K D-E P I}$ estimated glomerular filtration rate calculated by the Chronic Kidney Disease Epidemiology Collaboration equation, ERT enzyme replacement therapy, $m G F R_{\text {iohexol }}$ estimated glomerular filtration rate determined from clearance of iohexol, N/A not applicable, $S D$ standard deviation 


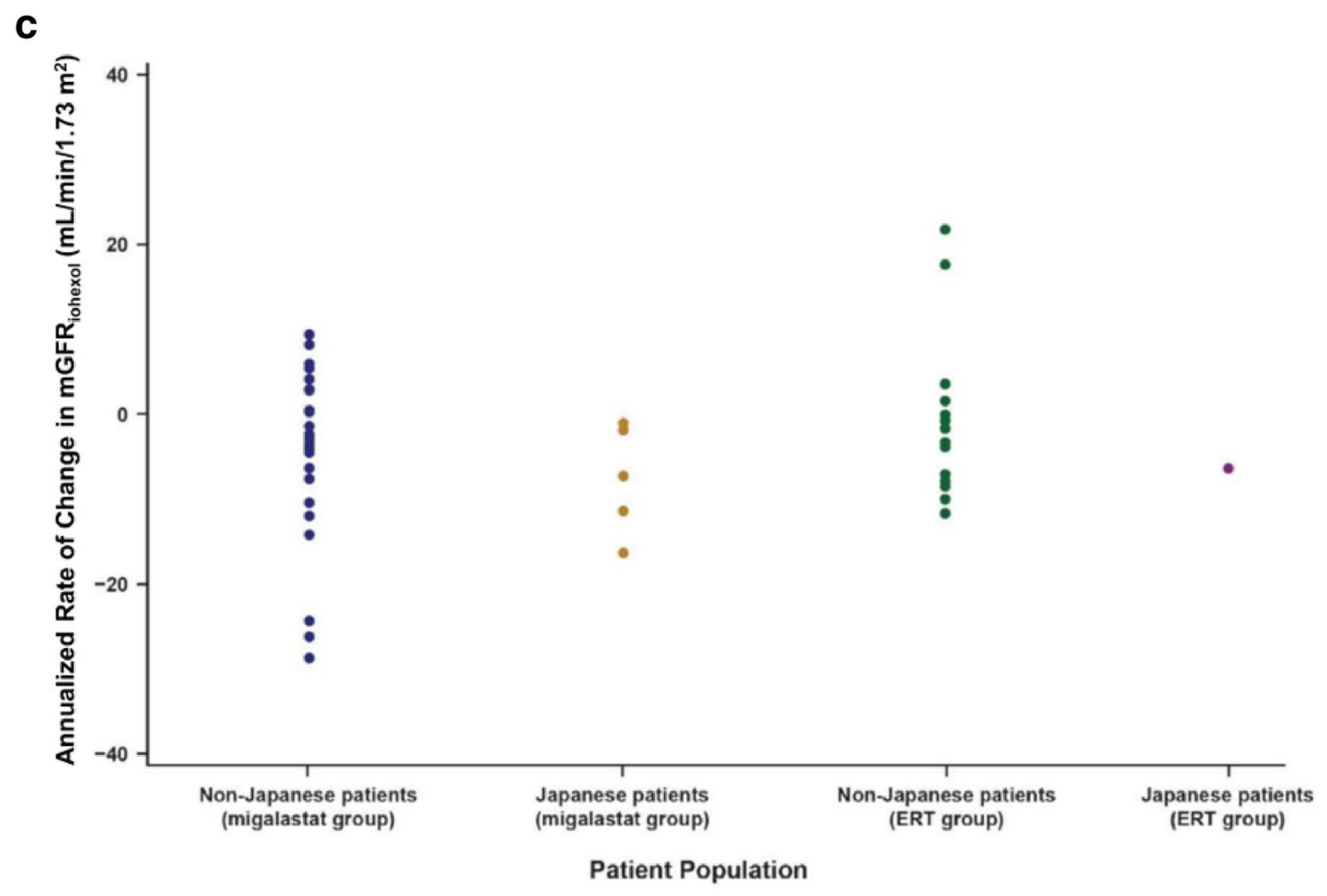

Fig. 2 (continued)

group (mean change, $-13.8 \mathrm{~g} / \mathrm{m}^{2} ; n=5$ ). The change in LVMi in the Japanese patients who received migalastat was greater than in the overall ATTRACT patient population $\left(-13.8 \mathrm{~g} / \mathrm{m}^{2}\right.$ vs $\left.-6.6 \mathrm{~g} / \mathrm{m}^{2}\right)$. Mean baseline LVMi was higher in the Japanese patient population than in the overall patient population $\left(111.3 \mathrm{~g} / \mathrm{m}^{2} \mathrm{vs} 97.5 \mathrm{~g} / \mathrm{m}^{2}\right)$. As in the overall patient population, Japanese patients who had baseline left ventricular hypertrophy showed a greater decrease of LVMi from baseline to month $18\left(-19.4 \mathrm{~g} / \mathrm{m}^{2}\right)$.

In the migalastat group $(n=5)$, the decrease in LVMi persisted to month 30 (Fig. 3). In the OLE study AT1001-042, the mean change of LVMi from baseline to month 42 was $-27.2 \mathrm{~g} / \mathrm{m}^{2}$ in 2 patients with echocardiographic data.

\section{Composite clinical outcomes}

In the overall patient population, the occurrence of combined clinical outcome (renal events, cardiac events, cerebrovascular events, and death) through month 18 was $29 \%$ in the migalastat group and $44 \%$ in the ERT group $(p=0.36)$. No deaths were reported during the 18-month open-label comparison period [18].

Among the Japanese patients, a renal event (i.e., > 33\% increase of 24-h urinary protein excretion compared with baseline and 24-h urinary protein excretion $\geq 300 \mathrm{mg}$ ) occurred in 1 of 5 patients in the migalastat group during the 18-month open-label comparison period, and a cardiac event (i.e., New York Heart Association class III/IV congestive heart failure) occurred in the 1 patient in the ERT group (Table 3).

\section{Plasma lyso-Gb 3 level}

In patients from the overall population with amenable $G L A$ mutations, the plasma lyso- $\mathrm{Gb}_{3}$ level remained low and stable after switching from ERT to migalastat [18]. In the Japanese patients who received migalastat $(n=5)$, the mean baseline plasma lyso- $\mathrm{Gb}_{3}$ level was $6.57 \mathrm{nmol} / \mathrm{L}$. As in the overall patient population, plasma lyso- $\mathrm{Gb}_{3} \mathrm{lev}$ els remained low and stable through month 18 during migalastat treatment in the Japanese patients. The mean change of plasma lyso- $\mathrm{Gb}_{3}$ from baseline to month 18 was $0.06 \mathrm{nmol} / \mathrm{L}$, which was within the $95 \% \mathrm{CI}$ of the mean change in the overall patient population $(95 \% \mathrm{CI}-0.30$ to 3.76 ).

In these 5 Japanese patients in the migalastat group, the mean plasma lyso- $\mathrm{Gb}_{3}$ level remained low and stable through month 30 (mean change from baseline to month $30,-0.04 \mathrm{nmol} / \mathrm{L} ; 95 \% \mathrm{CI}-1.80$ to 1.70 ). At month 30 , the plasma lyso- $\mathrm{Gb}_{3}$ level ranged from 3.30 to $13.67 \mathrm{nmol} / \mathrm{L}$ for men and from 2.11 to $4.19 \mathrm{nmol} / \mathrm{L}$ for women. 


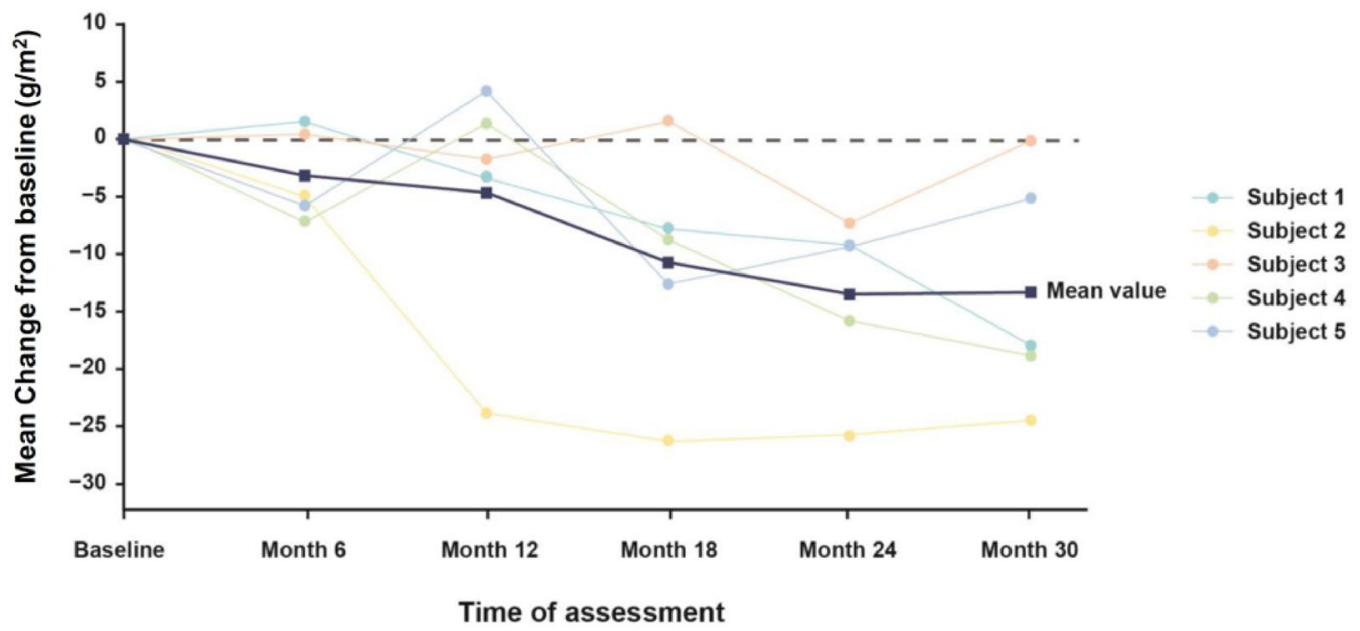

Mean change (SD) NA $\quad-3.2(3.9) \quad-4.7(11.1) \quad-10.7(10.1) \quad-13.5(7.6) \quad-13.3(10.2)$

Fig. 3 Change in LVMi $\left(\mathrm{g} / \mathrm{m}^{2}\right)$ from baseline to month 30 in Japanese patients who received migalastat. LVMi data are from 5 Japanese patients who were randomly assigned to migalastat in the ATT RACT study and then enrolled in AT1001-042 OLE study. Blue line

\section{Safety}

In the overall patient population, the frequency of AEs was 94\% in the migalastat group and 95\% in the ERT group [17]. The most frequently reported AEs in the migalastat group were nasopharyngitis (33\%) and headache (25\%). Most were mild or moderate. None of the patients discontinued the study because of AEs, and no treatment-related deaths or serious AEs were reported [18].

In the Japanese patient subpopulation, the frequency of AEs from baseline to month 30 was $100 \%$ in the migalastat group (5 of 5 patients) (Table 4). All AEs were mild or moderate. Nasopharyngitis was the only AE that occurred in 2 or more patients $(n=4,80 \%)$. No safety concerns were is mean change from baseline; remaining lines represent individual patient data. $L V M i$ left ventricular mass index, N/A not applicable, $O L E$ open-label extension, $S D$ standard deviation

reported in the OLE study AT1001-042. During the OLE period, nasopharyngitis was the only AE that occurred in 2 or more patients $(n=2,40 \%)$. None of the Japanese patients experienced headache at any point during the 2 studies. No clinically meaningful changes in vital signs, laboratory data, or physical findings were noted in the Japanese patient subpopulation.

\section{Discussion}

Migalastat is an oral agent that acts as a pharmacologic chaperone for $\alpha$-Gal A. In previous studies, migalastat provided clinical benefit with good tolerability in patients
Table 3 Number of events attributable to Fabry disease up to month 18 in the Japanese patient population and the overall patient population (mITT population) of the ATT RACT study

\begin{tabular}{llllll}
\hline Event, $n(\%)$ & \multicolumn{2}{l}{ Japanese } & & Overall \\
\cline { 2 - 3 } & Migalastat $(n=5)$ & ERT $(n=1)$ & & Migalastat $(n=34)$ & ERT $(n=18)$ \\
\hline Renal & $1(20.0)^{\mathrm{a}}$ & 0 & & $8(23.5)$ & $6(33.3)$ \\
Cardiac & 0 & $1(100.0)^{\mathrm{b}}$ & & $2(5.9)$ & $3(16.7)$ \\
Cerebrovascular & 0 & 0 & 0 & $1(5.6)$ \\
Death & 0 & 0 & & 0 & 0 \\
Total & $1(20.0)$ & $1(100.0)$ & & $10(29.4)$ & $8(44.4)$
\end{tabular}

ERT enzyme replacement therapy, $m I T T$ modified intention-to-treat population

Patients in the mITT population are those with amenable GLA mutations who were assessed for baseline and postbaseline efficacy measures

${ }^{a}>33 \%$ increase of 24-h urinary protein excretion compared with baseline and 24-h urinary protein excretion $\geq 300 \mathrm{mg}$

${ }^{\mathrm{b}}$ New York Heart Association class III/IV congestive heart failure 
Table 4 Adverse events in the Japanese patients randomly assigned to the migalastat group $(n=5)$

\begin{tabular}{lcc}
\hline & $\begin{array}{l}\text { Month 0-30 (ATT } \\
\text { RACT) }\end{array}$ & $\begin{array}{l}\text { After month 30 } \\
\text { (AT1001-042 OLE } \\
\text { study) }\end{array}$ \\
\hline $\begin{array}{l}\text { Number of AEs } \\
\text { Number of patients who experienced AEs, } n(\%)\end{array}$ & $\begin{array}{c}5(100.0) \\
\text { The most frequently reported AEs (reported in 2 or more Japanese patients), } n(\%)\end{array}$ \\
$\begin{array}{l}\text { Nasopharyngitis } \\
4(80.0)\end{array}$ & $4(80.0)$ \\
\hline
\end{tabular}

$A E$ adverse event, $O L E$ open-label extension with Fabry disease and amenable GLA mutations [18, 21]. In the phase III FACETS study, migalastat was administered to patients with Fabry disease who had not received ERT. Results showed a decrease in renal interstitial capillary GL-3 inclusions and plasma lyso- $\mathrm{Gb}_{3}$ level due to migalastat treatment, along with stabilization of renal function, a decrease in cardiac mass, and improvement in gastrointestinal symptoms [21]. In the phase III ATT RACT study, migalastat is a treatment option for ERTexperienced patients [18].

No racial differences have been reported with regard to Fabry disease, and its clinical phenotypes are likely similar among races $[2,6,22]$. Therefore, it is meaningful that this subanalysis of Japanese patients showed that the efficacy of migalastat was not affected by race; there were also no unexpected safety concerns. The pharmacokinetics of migalastat was similar in the Japanese patients and the overall ATT RACT population, suggesting that migalastat's therapeutic efficacy is not affected by intrinsic factors or race [17]. In addition, efficacy and safety of migalastat were comparable in the Japanese and the overall patient populations.

Regarding the pharmacodynamic effects of migalastat, male Japanese patients with Fabry disease showed an increase in leukocyte $\alpha$-Gal A activity, and the median change was within the range for the overall ATTRACT population. In Japanese patients with amenable mutations, migalastat treatment also stabilized renal function and decreased LVMi, as observed in the overall patient population. In addition, the plasma lyso- $\mathrm{Gb}_{3}$ level remained low and stable throughout migalastat treatment in the Japanese patients and in the overall ATTRACT population. Additionally, individual patient data in migalastat group (Table 2) showed that eGFR range was relatively wide which might be due to either varied eGFR level of patients at baseline or concomitant treatment such as ACEI/ARB received by patients. However, given the small sample size in this study, the definitive cause could not be determined.

In the AT1001-042 OLE study, the beneficial effects of migalastat on renal function and LVMi were maintained in the Japanese patients through month 48 and month 42 , respectively. In addition to demonstrating efficacy, migalastat was also safe and well tolerated by Japanese patients. The types and frequencies of AEs were similar in both the Japanese and the overall patient populations.

Only a few prospective studies have been performed in Japanese patients with Fabry disease, highlighting the importance of the current findings, despite the small sample size. A recently reported observational study conducted in 36 previously untreated Japanese patients showed that ERT can stabilize renal function and prevent deterioration of cardiac function [6]. The patients in this recent study were relatively young (mean age 27 and 45 years for men and women, respectively), whereas the Japanese patients in the ATTRACT study were older (mean age 55 years), had previously been treated with ERT for 12 months or more before enrollment, and had a high disease burden. Use of migalastat achieved favorable outcomes in patients previously treated with ERT in the ATT RACT study, which included an open-label comparison with ERT and an OLE period [18]. Therefore, these findings are important when considering treatment options for Japanese patients with Fabry disease.

The ATTRACT study was characterized by a prospective design and by enrolling typical Japanese patients with Fabry disease. However, given the small sample size of the Japanese patient population, the results should be interpreted with caution when considering migalastat therapy.

With the caveat of the aforementioned study limitations, migalastat seems effective for Japanese patients with Fabry disease who have amenable GLA mutations. In addition, migalastat demonstrated similar efficacy and safety in Japanese patients with Fabry disease who had previously been treated with ERT compared with the overall ATT RACT patient population. Therefore, our findings confirm the pharmacologic actions and efficacy of migalastat in Japanese patients.

Acknowledgements The authors thank all those who participated in this study, including the patients, their families, fabry patients advocacy organizations in Japan, and health care providers. The authors also thank Sally Mitchell and Dana Francis of ApotheCom (Yardley, PA, United States) provided writing and editing support. Medical writing support was provided by MedPro Clinical Research. 
Funding This study was funded by Amicus Therapeutics, Inc. Preparation of this paper was funded by Amicus Therapeutics, Inc. and Amicus Therapeutics K.K.

\section{Compliance with ethical standards}

Conflict of interest Employment: Nina Skuban, Jeffrey P. Castelli, Hjalmar Lagast, and Jay A. Barth (Amicus Therapeutics Inc.); stock ownership or options: Nina Skuban, Jeffrey P. Castelli, Hjalmar Lagast, and Jay A. Barth (Amicus Therapeutics Inc.); research grant: Toyo Ohashi (ARVOBIO Inc., Dainippon Sumitomo Pharma, Sanofi Genzyme, Amicus Therapeutics Inc.) and Noroi Sakai (Sanofi Genzyme, Dainippon Sumitomo Pharma); Honoraria: Toyo Ohashi (Dainippon Sumitomo Pharma) and Norio Sakai (Sanofi Genzyme, Dainippon Sumitomo Pharma). All other authors have declared that no conflict of interest exists.

Ethical approval All procedures performed in studies involving human participants were in accordance with the ethical standards of the institutional and/or national research committee (IRB approval number for AT1001-012 and AT1001-042: The Jikei University School of Medicine: 23-25(2845) and 26-07(2935); Osaka City University Graduate School of Medicine: 1688 and 1827; Osaka University Graduate School of Medicine: 124002 and 146062; Niigata University Graduate School of Medical and Dental Sciences: $\mathrm{CH} 23-017$ and CH26-013) at which the studies were conducted according to International Conference on Harmonisation/Good clinical Practice (ICH/GCP) and with the 1964 Helsinki declaration and its later amendments or comparable ethical standards.

Informed consent Informed consent was obtained from all participants included in the present study.

Open Access This article is licensed under a Creative Commons Attribution 4.0 International License, which permits use, sharing, adaptation, distribution and reproduction in any medium or format, as long as you give appropriate credit to the original author(s) and the source, provide a link to the Creative Commons licence, and indicate if changes were made. The images or other third party material in this article are included in the article's Creative Commons licence, unless indicated otherwise in a credit line to the material. If material is not included in the article's Creative Commons licence and your intended use is not permitted by statutory regulation or exceeds the permitted use, you will need to obtain permission directly from the copyright holder. To view a copy of this licence, visit http://creativecommons.org/licenses/by/4.0/.

\section{References}

1. Ishii S, Chang HH, Kawasaki K, et al. Mutant alpha-galactosidase A enzymes identified in Fabry disease patients with residual enzyme activity: biochemical characterization and restoration of normal intracellular processing by 1-deoxygalactonojirimycin. Biochem J. 2007;406(2):285-95.

2. El-Abassi R, Singhal D, England JD. Fabry's disease. J Neurol Sci. 2014;344(1-2):5-19.

3. Germain DP. Fabry disease. Orphanet J Rare Dis. 2010;5:30.

4. Inoue $\mathrm{T}$, Hattori $\mathrm{K}$, Ihara $\mathrm{K}$, Ishii A, Nakamura K, Hirose S. Newborn screening for Fabry disease in Japan: prevalence and genotypes of Fabry disease in a pilot study. J Hum Genet. 2013;58(8):548-52.

5. Schiffmann R, Ries M. Fabry disease: a disorder of childhood onset. Pediatr Neurol. 2016;64:10-20.
6. Tsuboi K, Yamamoto H. Efficacy and safety of enzyme-replacement-therapy with agalsidase alfa in 36 treatment-naive Fabry disease patients. BMC Pharmacol Toxicol. 2017;18(1):43.

7. Sakuraba Hitoshi, Togawa Tadayasu, Tsukimura Takahiro, et al. Plasma lyso-Gb3: a biomarker for monitoring fabry patients during enzyme replacement therapy. Clin Exp Nephrol. 2018;22(4):843-9.

8. Concolino D, Amico L, Cappellini MD, et al. Home infusion program with enzyme replacement therapy for Fabry disease: the experience of a large Italian collaborative group. Mol Genet Metab Rep. 2017;12:85-91.

9. Desnick RJ, Schuchman EH. Enzyme replacement therapy for lysosomal diseases: lessons from 20 years of experience and remaining challenges. Annu Rev Genomics Hum Genet. 2012;13:307-35.

10. Amicus Therapeutics UK Ltd. Galafold $123 \mathrm{mg}$ hard capsules (summary of product characteristics). Buckinghamshire: Amicus Therapeutics UK Ltd; 2019.

11. Ishii S. Pharmacological chaperone therapy for Fabry disease. Proc Jpn Acad Ser B Phys Biol Sci. 2012;88(1):18-30.

12. Yam GH, Zuber C, Roth J. A synthetic chaperone corrects the trafficking defect and disease phenotype in a protein misfolding disorder. FASEB J. 2005;19(1):12-8.

13. Benjamin ER, Della Valle MC, Wu X, et al. The validation of pharmacogenetics for the identification of Fabry patients to be treated with migalastat. Genet Med. 2017;19(4):430-8.

14. Fan JQ, Ishii S, Asano N, Suzuki Y. Accelerated transport and maturation of lysosomal alpha-galactosidase A in Fabry lymphoblasts by an enzyme inhibitor. Nat Med. 1999;5(1):112-5.

15. Amicus Therapeutics. Galafold (prescribing information). Cranbury: Amicus Therapeutics; 2018.

16. Amicus Therapeutics announces approval of Galafold ${ }^{\circledR}$ (Migalastat) for fabry disease in Japan: first amicus medicine and first oral precision medicine for fabry patients with an amenable mutation in Japan [press release]. GlobeNewswire, 2018. http:// ir.amicusrx.com/news-releases/news-release-details/amicus-thera peutics-announces-approval-galafoldr-migalastat. Published 22 March 2018. Accessed 4 June 2019.

17. Ino H, Takahashi N, Terao T, Mudd PN Jr, Hirama T. Pharmacokinetics, safety, and tolerability following single-dose migalastat hydrochloride (GR181413A/AT1001) in healthy male Japanese subjects. J Drug Assess. 2013;2(1):87-93.

18. Hughes DA, Nicholls K, Shankar SP, et al. Oral pharmacological chaperone migalastat compared with enzyme replacement therapy in Fabry disease: 18-month results from the randomised phase III ATTRACT study. J Med Genet. 2017;54(4):288-96.

19. Wu X, Katz E, Della Valle MC, et al. A pharmacogenetic approach to identify mutant forms of alpha-galactosidase A that respond to a pharmacological chaperone for Fabry disease. Hum Mutat. 2011;32(8):965-77.

20. European Medicines Agency. Supply shortage of Fabrazymeupdated treatment recommendations required for adult male patients [press release]. https://www.ema.europa.eu/en/news/suppl y-shortage-fabrazyme-updated-treatment-recommendations-requi red-adult-male-patients. Published 25 September 2009. Accessed 4 June 2019.

21. Germain DP, Hughes DA, Nicholls K, et al. Treatment of Fabry's disease with the pharmacologic chaperone migalastat. $\mathrm{N}$ Engl J Med. 2016;375(6):545-55.

22. Eng CM, Fletcher J, Wilcox WR, et al. Fabry disease: baseline medical characteristics of a cohort of 1765 males and females in the Fabry Registry. J Inherit Metab Dis. 2007;30(2):184-92.

Publisher's Note Springer Nature remains neutral with regard to jurisdictional claims in published maps and institutional affiliations. 